\title{
Neural Network based Vehicle Classification for Intelligent Traffic Control
}

\author{
Saeid Fazli ${ }^{1}$, Shahram Mohammadi ${ }^{2}$, Morteza Rahmani ${ }^{3}$ \\ 1,2,3 Electrical Engineering Department, Zanjan University, Zanjan, IRAN \\ Fazlieznu.ac.ir, Shahrameznu.ac.ir, Rahmanimeznu.ac.ir
}

\begin{abstract}
Nowadays, number of vehicles has been increased and traditional systems of traffic controlling couldn't be able to meet the needs that cause to emergence of Intelligent Traffic Controlling Systems. They improve controlling and urban management and increase confidence index in roads and highways. The goal of this article is vehicles classification base on neural networks. In this research, it has been used a immovable camera which is located in nearly close height of the road surface to detect and classify the vehicles. The algorithm that used is included two general phases; at first, we are obtaining mobile vehicles in the traffic situations by using some techniques included image processing and remove background of the images and performing edge detection and morphology operations. In the second phase, vehicles near the camera are selected and the specific features are processed and extracted. These features apply to the neural networks as a vector so the outputs determine type of vehicle. This presented model is able to classify the vehicles in three classes; heavy vehicles, light vehicles and motorcycles. Results demonstrate accuracy of the algorithm and its highly functional level.
\end{abstract}

\section{KEYWORDS}

traffic controlling, vehicle classification, camera, neural network, image processing.

\section{INTRODUCTION}

By developing of digital cameras and increasing of power in processing computer, application of this tools increased. So that in the most systems, it is used of these technologies. The systems of controlling traffic intelligently was one of the applications which tools account of most countries. By increasingly growth of vehicles and crossing them in the roads and highways and unresponsible of available fundamental of vehicle. The need to a automatic system is presented much more. The increase of demand for travelling in highways has reached to a explosive growth in recent years. Building road and spreading fundamentals of vehicle is not only way and should apply some new technologies for controlling traffic.

A way of controlling by video has been pay attention in parts of view computer. Nowadays In most of the societies, It,s used of this intelligent system for controlling traffic and obtaining those parameters of transportation. The using of systems of based on computer vision has been accounted. It,s because of facility installation and high level of efficiency in controlling traffic $[1,2,3]$. One of the most effective area in machine vision is processing into one mobile image. So that it can obtain a lot of features of mobile objects.

One of the most important and basic in auto controlling traffic are detection and classification. These process cause to extract a lot of parameters of traffic [2,4]. One of the hardest matter in 
computer vision is vehicle classification which need to a complicated algorithm and high accuracy. So far diverse works have considered for this classification. These process mostly are finding about separation and classify of vehicle from passengers. One of these ways is which Gupte et al [5]. is suggester. In this suggestive way it has been used of dimensions of vehicles for separation of cars and walked passengers. But It,s needed to more complicated for getting better answer. Ma et al. have presented a new way of classification that is used of modifiers SIFT [6]. But responsive of this system is still unknown. The method of detection and classification of vehicles with 3D was a way which Alex et al. suggester but It has own problems $[7,8]$. One of the greatest problem is modelling of all vehicles as a $3 \mathrm{D}$ that almost is not possible. Our suggestive method is a kind of neural network MLP that is used for vehicle classification.

\section{THE SUGGESTED ALGORITHM}

In this section we are analysing the algorithm which is used in this project. The algorithm includes two general phases that each has different parts. The first phase in algorithm includes background extraction and second includes finding passing vehicles and extracting the special features. These features have been applying to a neural network multilayer perceptron (MLP) and the classification of the vehicles has been being created as an output of neural network eventually. This process has been described as Figure (1) following:

Movies that used in this project have been provided from several highways in province ZanjanIran.

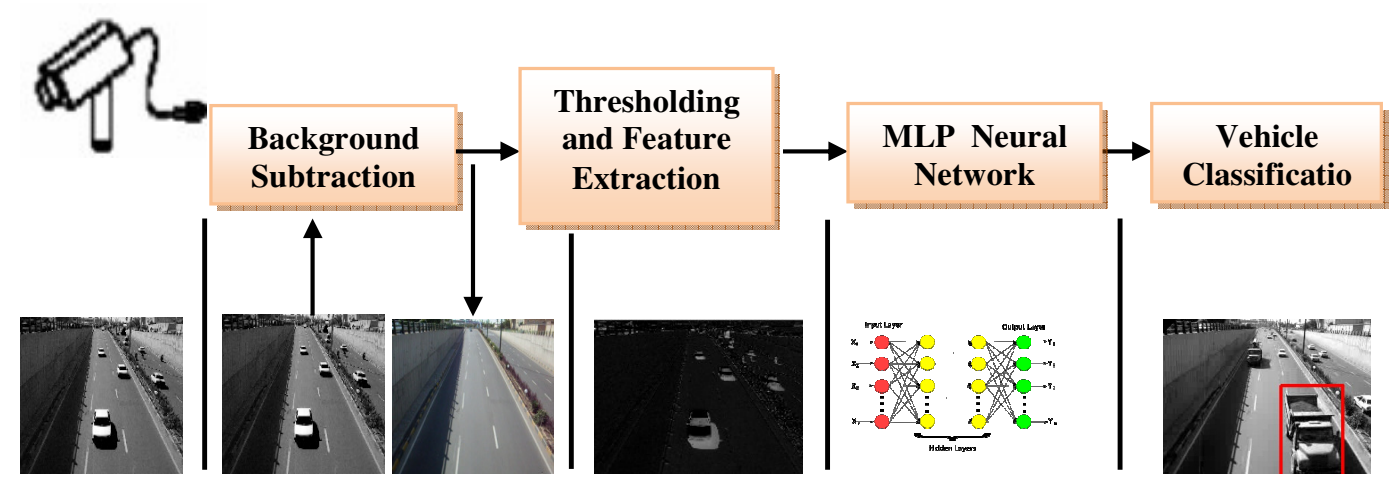

Figure 1. System overview

\subsection{Background Extraction}

To obtaining a background image, we are implementing following steps:

At first, we divide the input film obtained from the traffic scene into its forming frames and each frame is converting to gray level image. By using threshold of all columns and line of frame, if difference of pixels between 50 frames was smaller than a specific threshold, its value will be considered as intensity of light in the background. In Figure (2) X, Y axis shows size of the input image and position of each pixel has axis of $\mathrm{X}, \mathrm{Y}$ and also height shows amount of brightness intensity. 


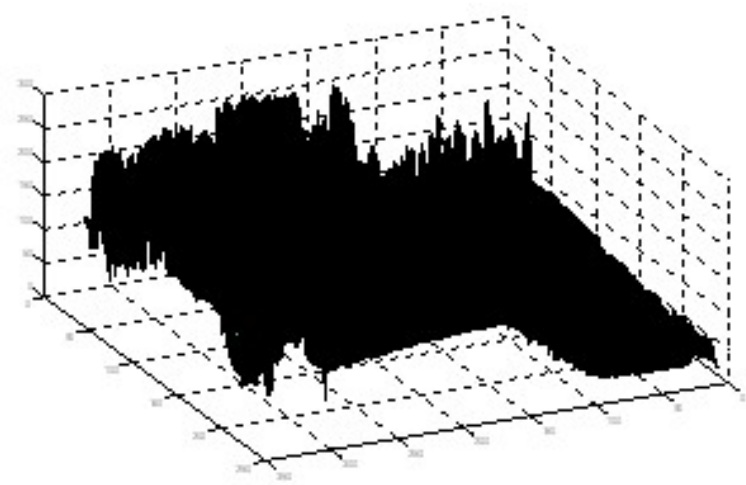

Figure 2. 3D drawing of background image

Now, It's gained some matrix that is input matrix as a background.

\subsection{Extracting vehicles from an image}

Extracting of vehicle is the most common way to obtain changes between two frames. For recognition the changes between two frames of an image, the $f\left(x, y, t_{i}\right)$ and $f\left(x, y, t_{j}\right)$ frames take in $t_{i}$ and $t_{j}$ times respectively, and comparison have be done for every pixels. For doing this, we are considering the background that extracted of previous step as a resource image which includes some constant items. Comparing of this image and the second one from the same traffic scene include vehicles, we can subtract invariant elements in these images that have no differences and maintain non-zero inputs that are equal to variant items of the images. Figure (3) shows images of the subtraction of two images taken in $t_{i}$ and $t_{j}$. subtracted as following: [1]

$$
d_{i j}(x, y)= \begin{cases}1 & \left|f\left(x, y, t_{i}\right)-f\left(x, y, t_{j}\right)\right|>T \\ 0 & \text { otherwise } .\end{cases}
$$

Witch $\mathrm{T}$ is threshold.

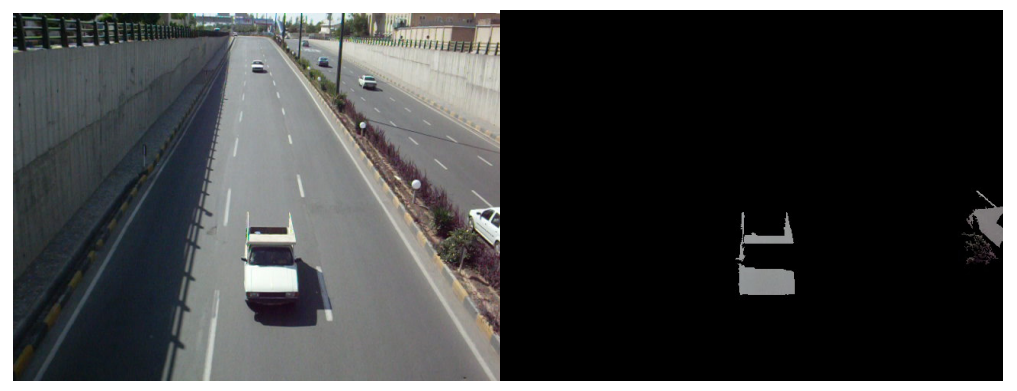

A

B

Figure 3. A) Original image B) Difference image from the original image

Now, It is used of method of approximation derive of image for recognizing of edge and then when ever of image that has gradient in pixels is that edge. Since It might charge the object into 
International Journal of Software Engineering \& Applications (IJSEA), Vol.3, No.3, May 2012

several piece after finding edge or by using of operator of dilation morphology of these pieces are linked together so it will be obtained a same object. As a result finding image will be labeled.

\subsection{Recognizing of vehicles}

This system is a form whence the vehicle is reached to the nearest distance of camera it can detect it, in this case it dosent need to any other previous vehicle in frame. So, we find those frames which put vehicles in the nearest distance to camera. Therefore, the process of detection begins and we are processing the image of the nearest vehicle.

Now, we are obtaining the features of the some special object in the image. There are six feature that including: length and width of the labelled objects, the area of object, in this case the obtaining object will have some small cavities and holes after the subtracting process of background. In this section, at first we are considering those cavities and holes and then obtaining the total area of object which is like a crick. An other feature of them refers to the real numbers of pixels in object. We need to use of two others features for better extracting vehicles. If we put a circle into the object so that it can cover it, we use of diameter of this circle for another feature. The last step is obtaining the ratio of distance between the centre of object and its length's main axis, we use it for extracting features variable. After extracting features, we'll make the vector for these features.

\subsection{Obtaining the output from the neural network}

After, that processing in the image and extracting of those features as a feature's vector to the neural network, so process of recognizing is done. The order of operation is this, at first we are saving the vector in the file excel which including six columns and each of them is presentation of a feature of object. We are specifying every kind of this feature's vector for our classification including three types: heavy vehicle, light vehicle and motorcycle. We are producing these data for neural network and then are defined the target vector for teaching neural network. The special neural network that is considered in this project is multilayer perceptron (MLP) with two hidden layers.

The output of neural network can recognize the type crossing vehicle which has shown in Figure (4). In this project we have classified the vehicles into 3 types as a heavy and light vehicles and motorcycle. At last the performance of the system was satisfied.

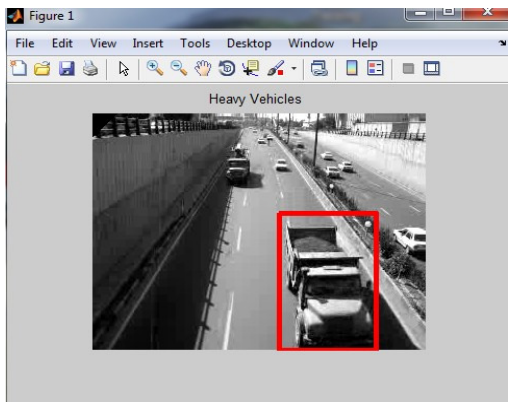

Figure 4. Result of the algorithm when crossing heavy vehicle 


\section{EXPERIMENTAL RESULTS}

In today society, the techniques of vehicle detection and classification based on visuality of camera for controlling traffic is the one the main programs and effective in the system of vehicle. Intelligent systems made the people to have ensured more than before. So, This system is conducting in the most countries. The designed system in this study is in the height almost higher than roods which has used for film taking of trafficking scene with a instant camera. It is able to detect and classify of crossing vehicle with minimum information. It's one of the most important factor in automatic controlling of traffic and finding a lot of details of traffic. It also helps to decrease of accidents. In addition to ability of vehicle detection and classification, it will be able to obtain the numbers of crossing cars in every time. The algorithm is done successfully. Figure (5) illustrates a part of results.

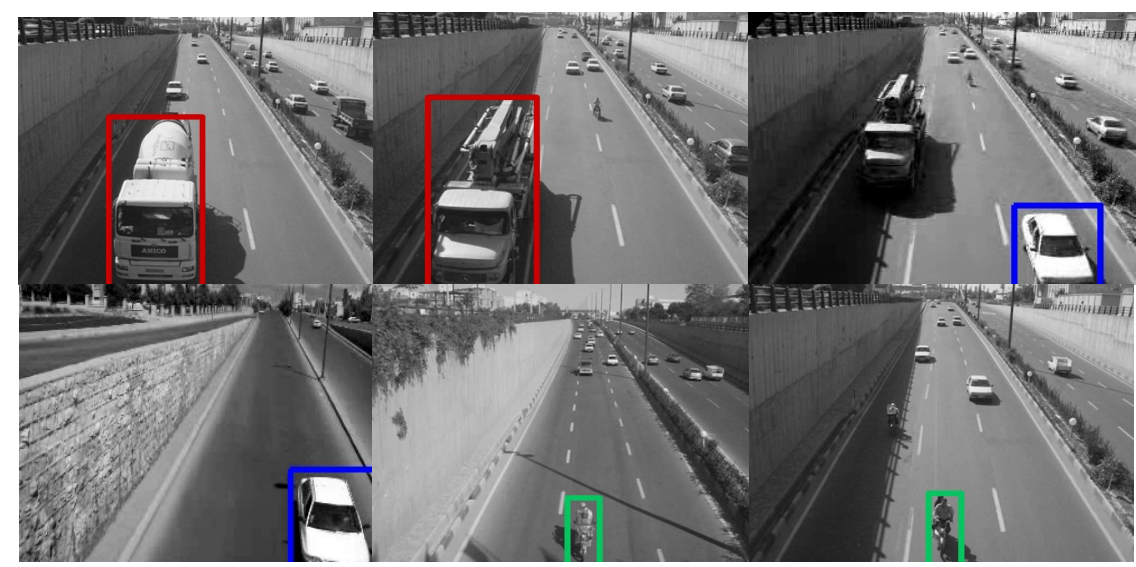

Figure 5. Results of the algorithm on some image frame

The outcome results verified the accuracy and high level of the performance of this method. So that it can detect and classify the special system with higher than $\% 90$ of crossing vehicle in the scenes of traffic. The finding results have been show in the following tables.

Table 1. Classification Results

\begin{tabular}{|c|c|c|c|c|}
\hline \multicolumn{1}{|c|}{} & $\begin{array}{c}\text { Heavy } \\
\text { vehicles }\end{array}$ & $\begin{array}{c}\text { Light } \\
\text { Vehicles }\end{array}$ & Motorcycle & $\begin{array}{c}\text { Total } \\
\text { vehicles }\end{array}$ \\
\hline $\begin{array}{c}\text { Number Of } \\
\text { Vehicle }\end{array}$ & 21 & 128 & 33 & 182 \\
\hline $\begin{array}{c}\text { Number Of } \\
\text { Detection }\end{array}$ & 17 & 119 & 28 & 164 \\
\hline $\begin{array}{c}\text { Classification } \\
\text { Rate }\end{array}$ & $81 \%$ & $93 \%$ & $85 \%$ & $90 \%$ \\
\hline
\end{tabular}


International Journal of Software Engineering \& Applications (IJSEA), Vol.3, No.3, May 2012

\section{CONCLUSION}

In this project, a traffic controlling system base on neural networks has been presented that it can recognize vehicles in a traffic scene and categorize it. Finding transportation parameters especially number and type of vehicles are one of the most important and applicable factors that cause to increase confidence index and reduce road crashes in roadways and highways. The camera that used in this project located in a place above the road surface. As an advantage of used method in design of algorithm is the camera don'ts need to calibration. After recognition of vehicles, they have been categorized in three classes. The results of testing of this method in several highways in Zanjan city were satisfying. In the future we can integrate whole of the system and make a network, then it is possible to categorize transportation vehicles base on real time.

\section{REFERENCES}

[1] Naixue Xiong, Jing He, Jong Hyuk Park, Donald Cooley, Yingshu Li. A Neural Network Based Vehicle Classification System for Pervasive Smart Road Security. Journal of Universal Computer Science, vol. 15, no. 5 (2009), 1119-1142

[2] N. K. Kanhere and S. T. Birchfield. Real-Time Incremental Segmentation and Tracking of Vehicles at Low Camera Angles Using Stable Features. IEEE Transactions on Intelligen Transportation Systems, 9(1):148-160, March 2008.

[3] N. K. Kanhere, S. T. Birchfield, W. A. Sarasua, and S. Khoeini. Traffic Monitoring of Motorcycles During Special Events Using Video Detection. Transportation Research Record: Journal of the Transportation Research Board, 2010.

[4] Jin-Cyuan Lai, and Shih-Shinh Huang, and Chien-Cheng Tseng. Image-based vehicle tracking and classification on the highway. Green Circuits and Systems (ICGCS), 2010 International Conference on, NO. 21, June 2010.

[5] S. Gupte, O. Masoud, R. F. K. Martin, and N. P. Papanikolopoulos. Detection and Classification of Vehicles. IEEE Transactions on Intelligent Transportation Systems, 3(1), 2002, 37-47.

[6] X. Ma, W. E. L. Grimson. Edge-based rich representation for vehicle classification. Proc. of the International Conference on Computer Vision, 2006, 1185-1192.

[7] Amol Ambardekar, Mircea Nicolescu, and George Bebis. Efficient Vehicle Tracking and Classification for an Automated Traffic Surveillance System. Department of Computer Science and Engineering University of Nevada, Reno U.S.A., Aug 2008.

[8] Limin Xia. Vehicle Recognition Using Boosting Neural Network Classifiers. Proceedings of the 6th World Congress on Intelligent Control and Automation, June 21 - 23, 2006, Dalian, China. 